Network Working Group

Request for Comments: 4167

Category: Informational
A. Lindem

Cisco Systems, Inc October 2005

\title{
Graceful OSPF Restart Implementation Report
}

Status of This Memo

This memo provides information for the Internet community. It does not specify an Internet standard of any kind. Distribution of this memo is unlimited.

Copyright Notice

Copyright (C) The Internet Society (2005).

Abstract

Graceful OSPF Restart, as specified in RFC 3623, provides a mechanism whereby an OSPF router can stay on the forwarding path even as its OSPF software is restarted. This document provides an implementation report for this extension to the base OSPF protocol.

Table of Contents

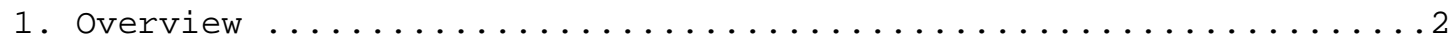

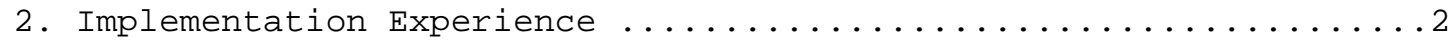

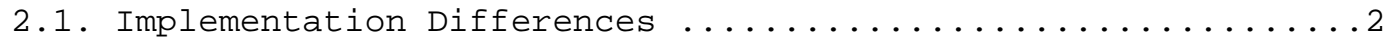

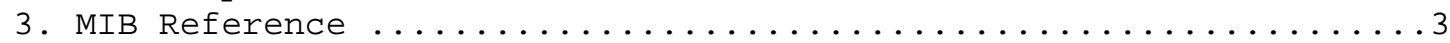

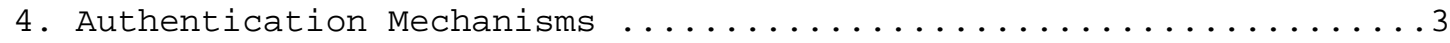

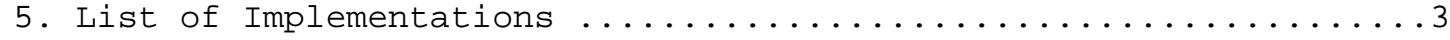

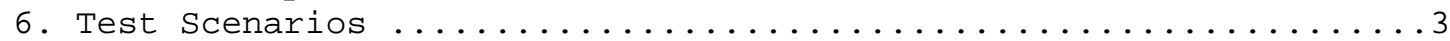

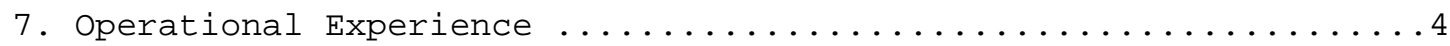

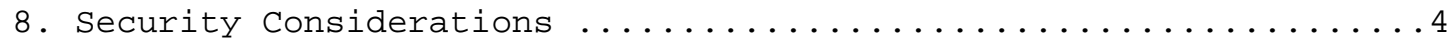

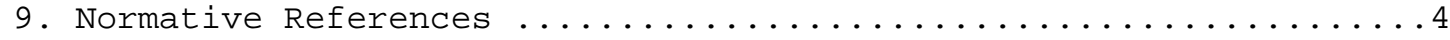

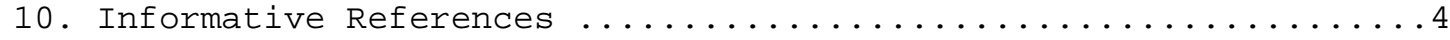

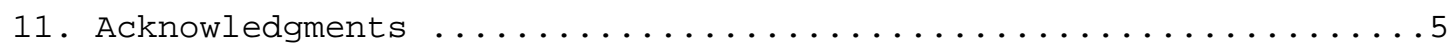




\section{Overview}

Today, many Internet routers implement a separation of control and forwarding functions. Certain processors are dedicated to control and management tasks such as OSPF routing, while other processors perform the data forwarding tasks. This separation creates the possibility of maintaining a router's data forwarding capability while the router's control software is restarted/reloaded. For the OSPF protocol [OSPF], the protocol mechanisms necessary to accomplish this are described in Graceful OSPF Restart [GRACE].

This document satisfies the RFC 1264 [CRITERIA] requirement for a report on implementation experience for Graceful OSPF Restart. Section 2 of this document contains the results of an implementation survey. It also documents implementation differences between the vendors responding to the survey. Section 3 contains a MIB reference. Section 4 provide an authentication reference. Section 5 simply refers to the implementations listed in section 2. Section 6 includes a minimal set of test scenarios. Finally, section 7 includes a disclaimer with respect to operational experience.

\section{Implementation Experience}

Eleven vendors have implemented graceful OSPF and have completed the implementation survey. These include Redback, Juniper, Motorola Computer Group (formerly Netplane Systems), Mahi Networks, Nexthop technologies, Forcelo Networks, Procket, Alcatel, Laurel Networks, DCL (Data Connection Limited), and Ericsson. All have implemented restart from the perspective of both a restarting and helper router. All but one vendor implemented both planned and unplanned restart. All implementations are original. Seven successfully tested interoperability with Juniper. Juniper successfully tested interoperability with Forcelo Networks. One vendor tested with John Moy's GNU Public License implementation [OSPFD]. Two vendors had not tested interoperability at the time of the survey.

\subsection{Implementation Differences}

The first difference was whether strict LSA checking was implemented and, if so, whether it was configurable. In the context of graceful OSPF restart, strict LSA checking indicates whether a changed LSA will result in the termination of graceful restart by a helping router. Four vendors made it configurable (three defaulted it to enabled and one to disabled), another made it a compile option (shipping with strict LSA checking disabled), another didn't implement it at all, and five implemented strict LSA checking with no configuration option to disable it. 
The second was whether a received grace LSA would be taken to apply only to the adjacency on which it was received or to all adjacencies with the restarting router. This is a rather subtle difference since it only applies to helping and restarting routers with more than one full adjacency at the time of restart. Eight vendors implemented the option of the received grace LSA only applying to the adjacency on which it was received. Three vendors applied the grace LSA to all adjacencies with the grace LSA originator (i.e., the restarting router).

The final difference was in whether additional extensions were implemented to accommodate other features such as protocol redistribution or interaction with MPLS VPNs [VPN]. Five vendors implemented extensions and six did not. It should be noted that such extensions are beyond the scope of Graceful OSPF Restart [GRACE].

\section{MIB Reference}

MIB objects for the Graceful OSPF Restart have been added to the OSPF Version 2 Management Information Base [OSPFMIB]. Additions include:

- Objects ospfRestartsupport, ospfRestartInterval, ospfRestartAge, ospfRestartExitReason, and ospfRestartStrictLsaChecking to ospfGeneralGroup.

- Objects ospenbrRestartHelperStatus, ospfNbrRestartHelperAge, and ospenbrRestartHelperExitReason to ospfNbrEntry.

- Objects ospfVirtNbrRestartHelperstatus, ospfVirtNbrRestartHelperAge, and ospfVirtNbrRestartHelperExitReason to ospfVirtNbrEntry.

4. Authentication Mechanisms

The authentication mechanisms are the same as those implemented by the base OSPF protocol [OSPF].

5. List of Implementations

Refer to section 2 .

6. Test Scenarios

A router implementing graceful restart should test, at a minimum, the following scenarios as both a restarting and helping router. For all scenarios, monitoring data plane traffic may be used to ensure that the restart is non-disruptive: 
1. Operation over a broadcast network.

2. Operation over a P2P network.

3. Operation over a virtual link.

4. Operation using OSPF MD5 authentication.

5. Early graceful restart termination when an LSA inconsistency is detected.

6. Early graceful restart termination when a flooded LSA changes (if implemented).

7. Operational Experience

Since OSPF graceful restart is configurable, it is difficult to gage operational experience at this juncture. However, multiple service providers have tested and evaluated it.

8. Security Considerations

This document does not discuss implementation and interoperability aspects of the security mechanisms in great detail, as no new security mechanisms are introduced with Graceful OSPF Restart. Security considerations for the OSPF protocol are included in RFC 2328 [OSPF]. Security considerations for Graceful OSPF Restart are included in [GRACE].

9. Normative References

[OSPF] Moy, J., "OSPF Version 2", STD 54, RFC 2328, April 1998.

[GRACE] Moy, J., Pillay-Esnault, P., and A. Lindem, "Graceful OSPF Restart", RFC 3623, November 2003.

[CRITERIA] Hinden, R., "Internet Engineering Task Force Internet Routing Protocol Standardization Criteria", RFC 1264, October 1991 .

10. Informative References

[VPN] Rosen, E. and Y Rekhter, "BGP/MPLS IP VPNs", Work in Progress, September 2003.

[OSPFD] Moy, J., "OSPF Complete Implementation", Addison-Wesley, 1991, ISBN 0-201-30966-1 
[OSPFMIB] Joyal, D., et al, "OSPF Version 2 Management Information Base", Work in Progress, December 2003.

11. Acknowledgments

The author wishes to acknowledge the individuals/vendors who have completed the implementation survey.

- Anand Oswal (Redback Networks)

- Padma Pillay-Esnault (Juniper Networks)

- Vishwas Manral (Motorola Computer Group, formerly Netplane System).

- Sriganesh Kini (Mahi Networks)

- Jason Chen (Forcelo Networks)

- Daniel Gryniewicz (NextHop Technologies)

- Hasmit Grover (Procket Networks)

- Pramoda Nallur (Alcatel)

- Ardas Cilingiroglu (Laurel Networks)

- Philip Crocker (Data Connection Limited)

- Le-Vinh Hoang (Ericsson)

Author's Address

Acee Lindem

Cisco Systems, Inc

7025 Kit Creek Road

Research Triangle Park, NC 27709

USA

EMail: aceedcisco.com 
Full Copyright statement

Copyright (C) The Internet Society (2005).

This document is subject to the rights, licenses and restrictions contained in BCP 78, and except as set forth therein, the authors retain all their rights.

This document and the information contained herein are provided on an "AS IS" basis and THE CONTRIBUTOR, THE ORGANIZATION HE/SHE REPRESENTS OR IS SPONSORED BY (IF ANY), THE INTERNET SOCIETY AND THE INTERNET ENGINEERING TASK FORCE DISCLAIM ALL WARRANTIES, EXPRESS OR IMPLIED, INCLUDING BUT NOT LIMITED TO ANY WARRANTY THAT THE USE OF THE INFORMATION HEREIN WILL NOT INFRINGE ANY RIGHTS OR ANY IMPLIED WARRANTIES OF MERCHANTABILITY OR FITNESS FOR A PARTICULAR PURPOSE.

Intellectual Property

The IETF takes no position regarding the validity or scope of any Intellectual Property Rights or other rights that might be claimed to pertain to the implementation or use of the technology described in this document or the extent to which any license under such rights might or might not be available; nor does it represent that it has made any independent effort to identify any such rights. Information on the procedures with respect to rights in RFC documents can be found in BCP 78 and BCP 79 .

Copies of IPR disclosures made to the IETF Secretariat and any assurances of licenses to be made available, or the result of an attempt made to obtain a general license or permission for the use of such proprietary rights by implementers or users of this specification can be obtained from the IETF on-line IPR repository at http://www.ietf.org/ipr.

The IETF invites any interested party to bring to its attention any copyrights, patents or patent applications, or other proprietary rights that may cover technology that may be required to implement this standard. Please address the information to the IETF at ietfipreietf.org.

Acknowledgement

Funding for the RFC Editor function is currently provided by the Internet Society. 